\title{
Chromatically Unique Multibridge Graphs
}

\author{
F.M. Dong \\ Mathematics and Mathematics Education, National Institute of Education \\ Nanyang Technological University, Singapore 637616 \\ fmdong@nie.edu.sg \\ K.L. Teo, C.H.C. Little*, M. Hendy \\ Institute of Fundamental Sciences PN461, Massey University \\ Palmerston North, New Zealand \\ k.l.teo@massey.ac.nz, c.little@massey.ac.nz,m.hendy@massey.ac.nz
}

\section{K.M. Koh}

Department of Mathematics, National University of Singapore

Singapore 117543

matkohkm@nus.edu.sg

Submitted: Jul 28, 2003; Accepted: Dec 13, 2003; Published: Jan 23, 2004

MR Subject Classification: 05C15

\begin{abstract}
Let $\theta\left(a_{1}, a_{2}, \cdots, a_{k}\right)$ denote the graph obtained by connecting two distinct vertices with $k$ independent paths of lengths $a_{1}, a_{2}, \cdots, a_{k}$ respectively. Assume that $2 \leq a_{1} \leq a_{2} \leq \cdots \leq a_{k}$. We prove that the graph $\theta\left(a_{1}, a_{2}, \cdots, a_{k}\right)$ is chromatically unique if $a_{k}<a_{1}+a_{2}$, and find examples showing that $\theta\left(a_{1}, a_{2}, \cdots, a_{k}\right)$ may not be chromatically unique if $a_{k}=a_{1}+a_{2}$.
\end{abstract}

Keywords: Chromatic polynomials, $\chi$-unique, $\chi$-closed, polygon-tree

\section{Introduction}

All graphs considered here are simple graphs. For a graph $G$, let $V(G), E(G), v(G), e(G)$, $g(G), P(G, \lambda)$ respectively be the vertex set, edge set, order, size, girth and chromatic polynomial of $G$. Two graphs $G$ and $H$ are chromatically equivalent (or simply $\chi$-equivalent),

\footnotetext{
${ }^{*}$ Corresponding author.
} 
symbolically denoted by $G \sim H$, if $P(G, \lambda)=P(H, \lambda)$. Note that if $H \sim G$, then $v(H)=v(G)$ and $e(H)=e(G)$. The chromatic equivalence class of $G$, denoted by $[G]$, is the set of graphs $H$ such that $H \sim G$. A graph $G$ is chromatically unique (or simply $\chi$-unique) if $[G]=\{G\}$. Whenever we talk about the chromaticity of a graph $G$, we are referring to questions about the chromatic equivalence class of $G$.

Let $k$ be an integer with $k \geq 2$ and let $a_{1}, a_{2}, \cdots, a_{k}$ be positive integers with $a_{i}+a_{j} \geq 3$ for all $i, j$ with $1 \leq i<j \leq k$. Let $\theta\left(a_{1}, a_{2}, \cdots, a_{k}\right)$ denote the graph obtained by connecting two distinct vertices with $k$ independent (internally disjoint) paths of lengths $a_{1}, a_{2}$, $\cdots, a_{k}$ respectively. The graph $\theta\left(a_{1}, a_{2}, \cdots, a_{k}\right)$ is called a multibridge (more specifically $k$-bridge) graph (see Figure 1).

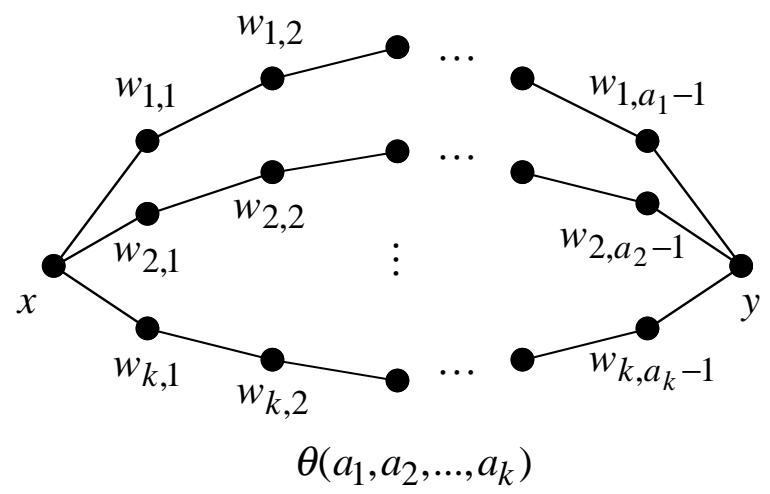

Figure 1

Given positive integers $a_{1}, a_{2}, \cdots, a_{k}$, where $k \geq 2$, what is a necessary and sufficient condition on $a_{1}, a_{2}, \ldots, a_{k}$ for $\theta\left(a_{1}, a_{2}, \ldots, a_{k}\right)$ to be chromatically unique? Many papers $[2,4,10,6,11,12,13,14]$ have been published on this problem, but it is still far from being completely solved $[8,9]$. In this paper, we shall solve this problem under the condition that $\max _{1 \leq i \leq k} a_{i} \leq \min _{1 \leq i<j \leq k}\left(a_{i}+a_{j}\right)$.

\section{Known results}

For two non-empty graphs $G$ and $H$, an edge-gluing of $G$ and $H$ is a graph obtained from $G$ and $H$ by identifying one edge of $G$ with one edge of $H$. For example, the graph $K_{4}-e$ (obtained from $K_{4}$ by deleting one edge) is an edge-gluing of $K_{3}$ and $K_{3}$. There are many edge-gluings of $G$ and $H$. Let $\mathcal{G}_{2}(G, H)$ denote the family of all edge-gluings of $G$ and $H$. Zykov [15] showed that any member of $\mathcal{G}_{2}(G, H)$ has chromatic polynomial

$$
P(G, \lambda) P(H, \lambda) /(\lambda(\lambda-1)) .
$$

Thus any two members in $\mathcal{G}_{2}(G, H)$ are $\chi$-equivalent.

For any integer $k \geq 2$ and non-empty graphs $G_{0}, G_{1}, \cdots, G_{k}$, we can recursively define

$$
\mathcal{G}_{2}\left(G_{0}, G_{1}, \cdots, G_{k}\right)=\bigcup_{\substack{0 \leq i \leq k \\ G^{\prime} \in \mathcal{G}_{2}\left(G_{0}, \cdots, G_{i-1}, G_{i+1}, \cdots, G_{k}\right)}} \mathcal{G}_{2}\left(G_{i}, G^{\prime}\right) .
$$


Each graph in $\mathcal{G}_{2}\left(G_{0}, G_{1}, \cdots, G_{k}\right)$ is also called an edge-gluing of $G_{0}, G_{1}, \cdots, G_{k}$. By (1), any two graphs in $\mathcal{G}_{2}\left(G_{0}, G_{1}, \cdots, G_{k}\right)$ are $\chi$-equivalent.

Let $C_{p}$ denote the cycle of order $p$. It was shown independently in [12] and [13] that if $G$ is $\chi$-equivalent to a graph in $\mathcal{G}_{2}\left(C_{i_{0}}, C_{i_{1}}, \cdots, C_{i_{k}}\right)$, then $G \in \mathcal{G}_{2}\left(C_{i_{0}}, C_{i_{1}}, \cdots, C_{i_{k}}\right)$. In other words, this family is a $\chi$-equivalence class.

For $k=2,3$, the graph $\theta\left(a_{1}, a_{2}, \cdots, a_{k}\right)$ is a cycle or a generalized $\theta$-graph respectively, and it is $\chi$-unique in both cases (see [10]). Assume therefore that $k \geq 4$. It is clear that if $a_{i}=1$ for some $i$, say $i=1$, then $\theta\left(a_{1}, a_{2}, \cdots, a_{k}\right)$ is a member of $\mathcal{G}_{2}\left(C_{a_{2}+1}, C_{a_{3}+1}, \cdots, C_{a_{k}+1}\right)$ and thus $\theta\left(a_{1}, a_{2}, \cdots, a_{k}\right)$ is not $\chi$-unique. Assume therefore that $a_{i} \geq 2$ for all $i$. For $k=4$, Chen, Bao and Ouyang [2] found that $\theta\left(a_{1}, a_{2}, a_{3}, a_{4}\right)$ may not be $\chi$-unique.

Theorem 2.1 ([2]) (a) Let $a_{1}, a_{2}, a_{3}, a_{4}$ be integers with $2 \leq a_{1} \leq a_{2} \leq a_{3} \leq a_{4}$. Then $\theta\left(a_{1}, a_{2}, a_{3}, a_{4}\right)$ is $\chi$-unique if and only if $\left(a_{1}, a_{2}, a_{3}, a_{4}\right) \neq(2, b, b+1, b+2)$ for any integer $b \geq 2$.

(b) The $\chi$-equivalence class of $\theta(2, b, b+1, b+2)$ is

$$
\{\theta(2, b, b+1, b+2)\} \cup \mathcal{G}_{2}\left(\theta(3, b, b+1), C_{b+2}\right) .
$$

Thus the problem of the chromaticity of $\theta\left(a_{1}, a_{2}, \cdots, a_{k}\right)$ has been completely settled for $k \leq 4$. For $k \geq 5$, we have

Theorem $2.2([14]) \quad$ For $k \geq 5, \theta\left(a_{1}, a_{2}, \cdots, a_{k}\right)$ is $\chi$-unique if $a_{i} \geq k-1$ for $i=$ $1,2, \cdots, k$.

Theorem $2.3([\mathbf{1 1}]) \quad$ Let $h \geq s+1 \geq 2$ or $s=h+1$. Then for $k \geq 5, \theta\left(a_{1}, a_{2}, \cdots, a_{k}\right)$ is $\chi$-unique if $a_{2}-1=a_{1}=h, a_{j}=h+s(j=3, \cdots, k-1), a_{k} \geq h+s$ and $a_{k} \notin$ $\{2 h, 2 h+s, 2 h+s-1\}$.

Theorems 2.2 and 2.3 do not include the case where $a_{1}=a_{2}=\cdots=a_{k}<k-1$.

Theorem 2.4 ([4], [6] and [13]) $\quad \theta\left(a_{1}, a_{2}, \cdots, a_{k}\right)$ is $\chi$-unique if $k \geq 2$ and $a_{1}=a_{2}=$ $\cdots=a_{k} \geq 2$.

\section{$3 \chi$-closed families of g.p. trees}

A $k$-polygon tree is a graph obtained by edge-gluing a collection of $k$ cycles successively, i.e., a graph in $\mathcal{G}_{2}\left(C_{i_{1}}, C_{i_{2}}, \cdots, C_{i_{k}}\right)$ for some integers $i_{1}, i_{2}, \cdots, i_{k}$ with $i_{j} \geq 3$ for all $j=1,2, \cdots, k$. A polygon-tree is a $k$-polygon tree for some integer $k$ with $k \geq 1$. A graph is called a generalized polygon tree (g.p. tree) if it is a subdivision of some polygon tree. 
Let $\mathcal{G P}$ denote the set of all g.p. trees. Dirac [3] and Duffin [5] proved independently that a 2-connected graph is a g.p. tree if and only if it contains no subdivision of $K_{4}$.

A family $\mathcal{S}$ of graphs is said to be chromatically closed (or simply $\chi$-closed) if $\bigcup_{G \in \mathcal{S}}[G]=$ $\mathcal{S}$. By using Dirac's and Duffin's result, Chao and Zhao [1] obtained the following result.

Theorem 3.1 ([1]) The set $\mathcal{G P}$ is $\chi$-closed.

The family $\mathcal{G P}$ can be partitioned further into $\chi$-closed subfamilies. Let $G \in \mathcal{G} \mathcal{P}$. A pair $\{x, y\}$ of non-adjacent vertices of $G$ is called a communication pair if there are at least three independent $x-y$ paths in $G$. Let $c(G)$ denote the number of communication pairs in $G$. For any integer $r \geq 1$, let $\mathcal{G} \mathcal{P}_{r}$ be the family of all g.p. trees $G$ with $c(G)=r$.

Theorem $3.2([\mathbf{1 3}]) \quad$ The family $\mathcal{G} \mathcal{P}_{r}$ is $\chi$-closed for every integer $r \geq 1$.

Let $G$ be a g.p. tree. We call a pair $\{x, y\}$ of vertices in $G$ a pre-communication pair of $G$ if there are at least three independent $x-y$ paths in $G$. If $x$ and $y$ are non-adjacent, then $\{x, y\}$ is a communication pair. Assume that $c(G)=1$. Then $G$ is a subdivision of a $k$ polygon tree $H$ for some $k \geq 2$. It is clear that $G$ and $H$ have the same pre-communication pairs. But not every pre-communication pair in $H$ is a communication pair. Since $c(G)=$ 1, only one pre-communication pair in $H$ is transformed into a communication pair in $G$. If $G$ has only one pre-communication pair, then $G$ is a multibridge graph. Otherwise, $G$ is an edge-gluing of a multibridge graph and some cycles. Therefore

$$
\mathcal{G} \mathcal{P}_{1}=\bigcup_{k \geq 3} \bigcup_{\substack{3 \leq t \leq k \\ b_{1}, b_{2}, \cdots, b_{k} \geq 2}} \mathcal{G}_{2}\left(\theta\left(b_{1}, b_{2}, \cdots, b_{t}\right), C_{b_{t+1}+1}, \cdots, C_{b_{k}+1}\right) .
$$

Hence we have

Lemma 3.1 Let $a_{i} \geq 2$ for $i=1,2, \cdots, k$, where $k \geq 3$. If $H \sim \theta\left(a_{1}, a_{2}, \cdots, a_{k}\right)$, then $H$ is either a $k$-bridge graph $\theta\left(b_{1}, \cdots, b_{k}\right)$ with $b_{i} \geq 2$ for all $i$ or an edge-gluing of a $t$-bridge graph $\theta\left(b_{1}, \cdots, b_{t}\right)$ with $b_{i} \geq 2$ for all $i$ and $k-t$ cycles for some integer $t$ with $3 \leq t \leq k-1$.

Note that for $G \in \mathcal{G}_{2}\left(\theta\left(b_{1}, b_{2}, \cdots, b_{t}\right), C_{b_{t+1}+1}, \cdots, C_{b_{k}+1}\right)$,

$$
e(G)=v(G)+k-2 \text {. }
$$

\section{A graph function}

For any graph $G$ and real number $\tau$, write

$$
\Psi(G, \tau)=(-1)^{1+e(G)}(1-\tau)^{e(G)-v(G)+1} P(G, 1-\tau) .
$$

Observe that $\Psi(G, \tau)=\Psi(H, \tau)$ if $G \sim H$. However, the converse is not true. For example, $\Psi(G, \tau)=\Psi\left(G \cup m K_{1}, \tau\right)$ but $G \nsim G \cup m K_{1}$ for any $m \geq 1$, where $G \cup m K_{1}$ is the graph obtained from $G$ by adding $m$ isolated vertices. However, we have 
Lemma 4.1 For graphs $G$ and $H$, if $G \sim H$, then $\Psi(G, \tau)=\Psi(H, \tau)$; if $v(G)=v(H)$ and $\Psi(G, \tau)=\Psi(H, \tau)$, then $G \sim H$.

Proof. We need to prove only the second assertion. Observe from (5) that $\Psi(G, \tau)$ is a polynomial in $\tau$ with degree $e(G)+1$. Thus $e(G)=e(H)$. Since $v(G)=v(H)$ and $\Psi(G, \tau)=\Psi(H, \tau)$, we have $P(G, 1-\tau)=P(H, 1-\tau)$. Therefore $G \sim H$.

Thus, by Lemma 4.1, for any graph $G,[G]$ is the set of graphs $H$ such that $v(H)=v(G)$ and $\Psi(H, \tau)=\Psi(G, \tau)$. In this paper, we shall use this property to study the chromaticity of $\theta\left(a_{1}, a_{2}, \cdots, a_{k}\right)$. We first derive an expression for $\Psi\left(\theta\left(a_{1}, a_{2}, \cdots, a_{k}\right), \tau\right)$.

The following lemma is true even if $k=1$ or $a_{i}=1$ for some $i$.

Lemma 4.2 For positive integers $k, a_{1}, a_{2}, \cdots, a_{k}$,

$$
\Psi\left(\theta\left(a_{1}, a_{2}, \cdots, a_{k}\right), \tau\right)=\tau \prod_{i=1}^{k}\left(\tau^{a_{i}}-1\right)-\prod_{i=1}^{k}\left(\tau^{a_{i}}-\tau\right) .
$$

Proof. By the deletion-contraction formula for chromatic polynomials, it can be shown that

$$
\begin{aligned}
& P\left(\theta\left(a_{1}, a_{2}, \cdots, a_{k}\right), \lambda\right) \\
= & \frac{1}{\lambda^{k-1}(\lambda-1)^{k-1}} \prod_{i=1}^{k}\left((\lambda-1)^{a_{i}+1}+(-1)^{a_{i}+1}(\lambda-1)\right) \\
& +\frac{1}{\lambda^{k-1}} \prod_{i=1}^{k}\left((\lambda-1)^{a_{i}}+(-1)^{a_{i}}(\lambda-1)\right) .
\end{aligned}
$$

Let $\tau=1-\lambda$. Then

$$
\begin{aligned}
& (-1)^{1+a_{1}+a_{2}+\cdots+a_{k}}(1-\tau)^{k-1} P\left(\theta\left(a_{1}, a_{2}, \cdots, a_{k}\right), 1-\tau\right) \\
= & \tau \prod_{i=1}^{k}\left(\tau^{a_{i}}-1\right)-\prod_{i=1}^{k}\left(\tau^{a_{i}}-\tau\right) .
\end{aligned}
$$

Since $v(G)=2-k+\sum_{i=1}^{k} a_{i}$ and $e(G)=\sum_{i=1}^{k} a_{i}$, by definition of $\Psi(G, \tau),(6)$ is obtained.

Corollary 4.1 For positive integers $k, a_{1}, a_{2}, \cdots, a_{k}$,

$$
\begin{gathered}
\Psi\left(\theta\left(a_{1}, a_{2}, \cdots, a_{k}\right), \tau\right)=(-1)^{k}\left(\tau-\tau^{k}\right) \\
+\sum_{\substack{1 \leq r \leq k \\
1 \leq i_{1}<i_{2}<\cdots<i_{r} \leq k}}(-1)^{k-r}\left(\tau-\tau^{k-r}\right) \tau^{a_{i_{1}}+a_{i_{2}}+\cdots+a_{i_{r}}} .
\end{gathered}
$$


We are now going to find an expression for $\Psi(H, \tau)$ for any $H$ in

$$
\mathcal{G}_{2}\left(\theta\left(b_{1}, b_{2}, \cdots, b_{t}\right), C_{b_{t+1}+1}, \cdots, C_{b_{k}+1}\right) \text {. }
$$

Lemma 4.3 Let $G$ and $H$ be non-empty graphs, and $M \in \mathcal{G}_{2}(G, H)$. Then

$$
\Psi(M, \tau)=\Psi(G, \tau) \Psi(H, \tau) /((-\tau)(1-\tau))
$$

Proof. Since $v(M)=v(G)+v(H)-2, e(M)=e(G)+e(H)-1$ and

$$
P(M, \lambda)=P(G, \lambda) P(H, \lambda) /(\lambda(\lambda-1)),
$$

by (5), (8) is obtained.

Lemma 4.4 Let $k, t, b_{1}, b_{2}, \cdots, b_{k}$ be integers with $3 \leq t<k$ and $b_{i} \geq 1$ for $i=$ $1,2, \cdots, k$. If $H \in \mathcal{G}_{2}\left(\theta\left(b_{1}, b_{2}, \cdots, b_{t}\right), C_{b_{t+1}+1}, \cdots, C_{b_{k}+1}\right)$, then

$$
\Psi(H, \tau)=\tau \prod_{i=1}^{k}\left(\tau^{b_{i}}-1\right)-\prod_{i=1}^{t}\left(\tau^{b_{i}}-\tau\right) \prod_{i=t+1}^{k}\left(\tau^{b_{i}}-1\right) .
$$

Proof. By (5), we have $\Psi\left(C_{b_{i}+1}, \tau\right)=(-\tau)(1-\tau)\left(\tau^{b_{i}}-1\right)$. Thus by (6) and (8), (10) is obtained.

\section{$5 \quad \chi$-unique multibridge graphs}

By Lemma 4.2, we can prove that $\theta\left(a_{1}, a_{2}, \cdots, a_{k}\right) \cong \theta\left(b_{1}, b_{2}, \cdots, b_{k}\right)$ if $\theta\left(a_{1}, a_{2}, \cdots, a_{k}\right) \sim$ $\theta\left(b_{1}, b_{2}, \cdots, b_{k}\right)$.

Lemma 5.1 Let $a_{i}$ and $b_{i}$ be integers with $1 \leq a_{1} \leq a_{2} \leq \cdots \leq a_{k}$ and $1 \leq b_{1} \leq b_{2} \leq$ $\cdots \leq b_{k}$, where $k \geq 3$. If

$$
\theta\left(a_{1}, a_{2}, \cdots, a_{k}\right) \sim \theta\left(b_{1}, b_{2}, \cdots, b_{k}\right)
$$

then $b_{i}=a_{i}$ for $i=1,2, \cdots, k$.

Proof. By Lemma 4.1 and Corollary 4.1, we have

$$
\begin{aligned}
& \sum_{\substack{1 \leq r \leq k \\
1 \leq i_{1}<i_{2}<\cdots<i_{r} \leq k}}(-1)^{k-r}\left(\tau-\tau^{k-r}\right) \tau^{a_{i_{1}}+a_{i_{2}}+\cdots+a_{i_{r}}} \\
& =\sum_{\substack{1 \leq r \leq k \\
1 \leq i_{1}<i_{2}<\cdots<i_{r} \leq k}}(-1)^{k-r}\left(\tau-\tau^{k-r}\right) \tau^{b_{i_{1}}+b_{i_{2}}+\cdots+b_{i_{r}}} \text {, }
\end{aligned}
$$

after we cancel the terms $(-1)^{k}\left(\tau-\tau^{k}\right)$ from both sides. The terms with lowest power in both sides have powers $1+a_{1}$ and $1+b_{1}$ respectively. Hence $a_{1}=b_{1}$. 
Suppose that $a_{i}=b_{i}$ for $i=1, \cdots, m$ but $a_{m+1} \neq b_{m+1}$ for some integer $m$ with $1 \leq m \leq k-1$. Since $a_{i}=b_{i}$ for $i=1,2, \cdots, m$, by (12), we have

$$
\begin{aligned}
\sum_{\substack{1 \leq r \leq k \\
1 \leq i_{1}<i_{2}<\cdots<i_{r} \leq k \\
i_{r}>m}}(-1)^{k-r}\left(\tau-\tau^{k-r}\right) \tau^{a_{i_{1}}+a_{i_{2}}+\cdots+a_{i_{r}}} \\
=\sum_{\substack{1 \leq r \leq k \\
1 \leq i_{1}<i_{2}<\cdots<i_{r} \leq k \\
i_{r}>m}}(-1)^{k-r}\left(\tau-\tau^{k-r}\right) \tau^{b_{i_{1}}+b_{i_{2}}+\cdots+b_{i_{r}}} .
\end{aligned}
$$

The terms with lowest power in both sides of (13) have powers $1+a_{m+1}$ and $1+b_{m+1}$ respectively. Hence $a_{m+1}=b_{m+1}$, a contradiction. Therefore $b_{i}=a_{i}$ for $i=1,2, \cdots, k$.

Let $a_{i}$ be an integer with $a_{i} \geq 2$ for $i=1,2, \cdots, k$ and suppose that $a_{1} \leq a_{2} \leq \cdots \leq a_{k}$. We shall show that $\theta\left(a_{1}, a_{2}, \cdots, a_{k}\right)$ is $\chi$-unique if $a_{k}<a_{1}+a_{2}$. It is well known (see [8]) that

Lemma 5.2 If $G \sim H$, then $g(G)=g(H)$.

Theorem 5.1 If $2 \leq a_{1} \leq a_{2} \leq \cdots \leq a_{k}<a_{1}+a_{2}$, where $k \geq 3$, then $\theta\left(a_{1}, a_{2}, \cdots, a_{k}\right)$ is chromatically unique.

Proof. By Theorem 2.2, we may assume that $a_{1} \leq k-2$.

By Lemmas 3.1 and 5.1, it suffices to show that $\theta\left(a_{1}, a_{2}, \cdots, a_{k}\right) \nsim H$ for any graph $H \in \mathcal{G}_{2}\left(\theta\left(b_{1}, b_{2}, \cdots, b_{t}\right), C_{b_{t+1}+1}, \cdots, C_{b_{k}+1}\right)$, where $t$ and $b_{i}$ are integers with $3 \leq t<k$ and $b_{i} \geq 2$ for $i=1,2, \cdots, k$. We may assume that $b_{1} \leq b_{2} \leq \cdots \leq b_{t}$ and $b_{t+1} \leq \cdots \leq b_{k}$.

Suppose that $H \sim \theta\left(a_{1}, a_{2}, \cdots, a_{k}\right)$. The girth of $\theta\left(a_{1}, a_{2}, \cdots, a_{k}\right)$ is $a_{1}+a_{2}$. Since

$$
g(H)=\min \left\{\min _{1 \leq i<j \leq t}\left(b_{i}+b_{j}\right), \min _{t+1 \leq i \leq k}\left(b_{i}+1\right)\right\}
$$

by Lemma 5.2 , we have $g(H)=a_{1}+a_{2}$ and

$$
\begin{cases}b_{i}+b_{j} \geq a_{1}+a_{2}, & 1 \leq i<j \leq t, \\ b_{i} \geq a_{1}+a_{2}-1, & t+1 \leq i \leq k\end{cases}
$$

As $e(H)=e\left(\theta\left(a_{1}, a_{2}, \cdots, a_{k}\right)\right)$, we have

$$
a_{1}+a_{2}+\cdots+a_{k}=b_{1}+b_{2}+\cdots+b_{k} .
$$

By Lemma 4.1, (6) and (10), we have

$$
\tau \prod_{i=1}^{k}\left(\tau^{a_{i}}-1\right)-\prod_{i=1}^{k}\left(\tau^{a_{i}}-\tau\right)=\tau \prod_{i=1}^{k}\left(\tau^{b_{i}}-1\right)-\prod_{i=1}^{t}\left(\tau^{b_{i}}-\tau\right) \prod_{i=t+1}^{k}\left(\tau^{b_{i}}-1\right) .
$$


We expand both sides of (17), delete $(-1)^{k} \tau$ from them and keep only the terms with powers at most $a_{1}+a_{2}$. Since $a_{i}+a_{j} \geq a_{1}+a_{2}$ and $b_{i}+b_{j} \geq a_{1}+a_{2}$ for all $i, j$ with $1 \leq i<j \leq k$, we have

$$
\begin{aligned}
& (-1)^{k-1} \sum_{i=1}^{k} \tau^{a_{i}+1}+(-1)^{k-1} \tau^{k}+(-1)^{k} \sum_{i=1}^{k} \tau^{k-1+a_{i}} \\
\equiv & (-1)^{k-1} \sum_{i=1}^{k} \tau^{b_{i}+1}+(-1)^{k-1} \tau^{t}+(-1)^{k} \sum_{i=1}^{t} \tau^{b_{i}+t-1} \\
& +(-1)^{k} \sum_{i=t+1}^{k} \tau^{b_{i}+t} \quad\left(\bmod \tau^{a_{1}+a_{2}+1}\right) .
\end{aligned}
$$

Observe that $b_{i}+t>a_{1}+a_{2}$ for $t+1 \leq i \leq k$ and $k-1+a_{i}>a_{1}+a_{2}$ for $2 \leq i \leq k$. Thus

$$
\begin{aligned}
& (-1)^{k-1} \sum_{i=1}^{k} \tau^{a_{i}+1}+(-1)^{k-1} \tau^{k}+(-1)^{k} \tau^{k-1+a_{1}} \\
\equiv & (-1)^{k-1} \sum_{i=1}^{k} \tau^{b_{i}+1}+(-1)^{k-1} \tau^{t}+(-1)^{k} \sum_{i=1}^{t} \tau^{b_{i}+t-1} \quad\left(\bmod \tau^{a_{1}+a_{2}+1}\right) .
\end{aligned}
$$

Hence

$$
\sum_{i=1}^{k} \tau^{a_{i}+1}+\tau^{k}+\sum_{i=1}^{t} \tau^{b_{i}+t-1} \equiv \sum_{i=1}^{k} \tau^{b_{i}+1}+\tau^{t}+\tau^{k-1+a_{1}} \quad\left(\bmod \tau^{a_{1}+a_{2}+1}\right) .
$$

Since $t \geq 3$, we have $b_{i}+t-1>a_{1}+a_{2}$ for $i \geq t+1$. If $b_{2}+t-1 \leq a_{1}+a_{2}$, then since $k-1+a_{1}>k$, the left side of (19) contains more terms with powers at most $a_{1}+a_{2}$ than does the right side, a contradiction. Hence $b_{i}+t-1>a_{1}+a_{2}$ for $2 \leq i \leq t$. Therefore

$$
\sum_{i=1}^{k} \tau^{a_{i}+1}+\tau^{k}+\tau^{b_{1}+t-1} \equiv \sum_{i=1}^{k} \tau^{b_{i}+1}+\tau^{t}+\tau^{k-1+a_{1}} \quad\left(\bmod \tau^{a_{1}+a_{2}+1}\right) .
$$

Note that $t \leq a_{1}+a_{2}$; otherwise, since $k>t$ and $a_{1}, b_{1} \geq 2,(20)$ becomes

$$
\sum_{i=1}^{k} \tau^{a_{i}+1}=\sum_{i=1}^{k} \tau^{b_{i}+1}
$$

which implies the equality of the multisets $\left\{a_{1}, a_{2}, \ldots, a_{k}\right\}$ and $\left\{b_{1}, b_{2}, \ldots, b_{k}\right\}$ in contradiction to (17).

Claim 1: There are no $i, j$ such that

$$
\left\{b_{1}, \cdots, b_{i-1}, b_{i+1}, \cdots, b_{k}\right\}=\left\{a_{1}, \cdots, a_{j-1}, a_{j+1}, \cdots, a_{k}\right\}
$$

as multisets.

Otherwise, by (16), $\left\{b_{1}, \cdots, b_{k}\right\}=\left\{a_{1}, \cdots, a_{k}\right\}$ as multisets, which leads to a contradiction by (17). 
Claim 2: $a_{2} \geq k-1$.

If $a_{2}<k-1$, then $a_{1}+k-1>a_{1}+a_{2}$. But $a_{i}+1 \leq a_{1}+a_{2}$ for $1 \leq i \leq k$. So, by (20), the multiset $\left\{a_{1}, \cdots, a_{j-1}, a_{j+1}, \cdots, a_{k}\right\}$ is a subset of the multiset $\left\{b_{1}, \cdots, b_{k}\right\}$ for some $j$ with $1 \leq j \leq k$, which contradicts Claim 1 .

Claim 3: $a_{1}=t-1$.

Since $\tau^{t}$ is a term of the right side of (20), the left side also contains $\tau^{t}$. But $k>t$, $b_{1}+t-1>t$ and, by Claim $2, a_{i}+1 \geq k>t$ for $i \geq 2$. Therefore $a_{1}+1=t$.

By Claim 3, (20) is simplified to

$$
\sum_{i=2}^{k} \tau^{a_{i}+1}+\tau^{k}+\tau^{b_{1}+t-1} \equiv \sum_{i=1}^{k} \tau^{b_{i}+1}+\tau^{k-1+a_{1}} \quad\left(\bmod \tau^{a_{1}+a_{2}+1}\right) .
$$

Claim 4: $b_{1}=k-1$.

Note that $k<a_{1}+a_{2}$, by Claim 2. As $\tau^{k}$ is a term of the left side of (21), the right side also contains this term. Thus $b_{i}+1=k$ for some $i$. If $i>t$, then by (15) and Claims 2 and 3 , we

$$
b_{i} \geq a_{1}+a_{2}-1 \geq k+t-3 \geq k,
$$

a contradiction. Thus $i \leq t$ and $b_{1} \leq b_{i}=k-1$. If $b_{1} \leq k-2$, then the right side of (21) has a term with power at most $k-1$. But the left side has no such term, a contradiction. Hence $b_{1}=k-1$.

By Claims 3 and 4, we have $\tau^{b_{1}+t-1}=\tau^{k-1+a_{1}}$. Thus (21) is further simplified to

$$
\sum_{i=2}^{k} \tau^{a_{i}+1}+\tau^{k} \equiv \sum_{i=1}^{k} \tau^{b_{i}+1} \quad\left(\bmod \tau^{a_{1}+a_{2}+1}\right) .
$$

Therefore the multiset $\left\{a_{2}, a_{3}, \cdots, a_{k}\right\}$ is a subset of the multiset $\left\{b_{1}, b_{2}, \cdots, b_{k}\right\}$, in contradiction to Claim 1.

Therefore $H \not \gamma\left(a_{1}, a_{2}, \cdots, a_{k}\right)$ and we conclude that $\theta\left(a_{1}, a_{2}, \cdots, a_{k}\right)$ is $\chi$-unique.

\section{$6 \quad \chi$-equivalent graphs}

In Section 5 , we proved that $\theta\left(a_{1}, a_{2}, \cdots, a_{k}\right)$ is $\chi$-unique if

$$
\max _{1 \leq i \leq k} a_{i}<\min _{1 \leq i<j \leq k}\left(a_{i}+a_{j}\right)
$$

Lemma 6.1 shows that, for any non-negative integer $n$, there exist examples where the graph $\theta\left(a_{1}, a_{2}, \ldots, a_{k}\right)$ is not $\chi$-unique and

$$
\max _{1 \leq i \leq k} a_{i}-\min _{1 \leq i<j \leq k}\left(a_{i}+a_{j}\right)=n .
$$

Lemma $6.1 \quad$ (i) $\theta(2,2,2,3,4) \sim H$ for every $H \in \mathcal{G}_{2}\left(\theta(2,2,3), C_{4}, C_{4}\right)$.

(ii) For $k \geq 4$ and $a \geq 2, \theta(k-2, a, a+1, \cdots, a+k-2) \sim H$ for every $H \in$ $\mathcal{G}_{2}\left(\theta(k-1, a, a+1, \cdots, a+k-3), C_{a+k-2}\right)$. 
(iii) For $k \geq 5, \theta(2,3, \cdots, k-1, k, k-3) \sim H$ for every graph $H$ in $\mathcal{G}_{2}(\theta(2,3, \cdots, k-$ 1), $\left.C_{k-1}, C_{k}\right)$.

It is straightforward to verify Lemma 6.1 by using Lemmas 4.1, 4.2 and 4.4.

It is natural to ask the following question: for which choices of $\left(a_{1}, a_{2}, \ldots, a_{k}\right)$ satisfying $k \geq 5$ and

$$
\max _{1 \leq i \leq k} a_{i}=\min _{1 \leq i<j \leq k}\left(a_{i}+a_{j}\right)
$$

is the graph $\theta\left(a_{1}, a_{2}, \ldots, a_{k}\right)$ chromatically unique? If $\theta\left(a_{1}, a_{2}, \cdots, a_{k}\right)$ is not $\chi$-unique, what is its $\chi$-equivalence class? The solution to this question will be given in another paper.

\section{References}

[1] C.Y. Chao and L.C. Zhao, Chromatic polynomials of a family of graphs, Ars. Combin. 15 (1983) 111-129.

[2] X.E. Chen, X.W. Bao and K.Z. Ouyang, Chromaticity of the graph $\theta(a, b, c, d), J$. Shaanxi Normal Univ. 20 (1992) 75-79.

[3] G.A. Dirac, A property of 4-chromatic graphs and some results on critical graphs, J. London Math. Soc. 27 (1952) 85-92.

[4] F.M. Dong, On chromatic uniqueness of two infinite families of graphs, J. Graph Theory 17 (1993) 387-392.

[5] R.J. Duffin, Topology of series-parallel networks, J. Math. Anal. Appl. 10 (1965) 303-318.

[6] K.M. Koh and C.P. Teo, Some results on chromatically unique graphs, Proc. Asian Math. Conf. (World Scientific, Singapore, 1990) 258-262.

[7] K.M. Koh and C.P. Teo, Chromaticity of series-parallel graphs, Discrete Math. 154 (1996) 289-295.

[8] K.M. Koh and K.L. Teo, The search for chromatically unique graphs, Graphs and Combinatorics 6 (1990) 259-285.

[9] K.M. Koh and K.L. Teo, The search for chromatically unique graphs -II, Discrete Math. 172 (1997) 59-78.

[10] B. Loerinc, Chromatic uniqueness of the generalized $\theta$-graphs, Discrete Math. 23 (1978) 313-316. 
[11] Y.H. Peng, On the chromatic coefficients of a graph and chromatic uniqueness of certain n-partition graphs, in: Combinatorics, Graph Theory, Algorithms and Applications (Beijing , 1993) (World Scientific, River Edge, NJ, 1994) 307-316.

[12] C.D. Wakelin and D.R. Woodall, Chromatic polynomials, polygon trees and outerplanar graphs, J. Graph Theory 16 (1992) 459-466.

[13] S.J. Xu, Classes of chromatically equivalent graphs and polygon trees, Discrete Math. 133 (1994) 267-278.

[14] S.J. Xu, J.J. Liu and Y.H. Peng, The chromaticity of $s$-bridge graphs and related graphs, Discrete Math. 135 (1994) 349-358.

[15] A.A. Zykov, On some properties of linear complexes, Amer. Math. Soc. Transl. No. 79 (1952); translated from Math. Sb. 24(66) (1949) 163-188. 